\title{
Urologische Onkologie - Fortschritte am Beispiel des Prostatakarzinoms
}

\author{
H. Rübben T. Otto
}

Urologische Klinik und Poliklinik, Universitätsklinikum Essen

Das Prostatakarzinom ist mit 13000 Tumortoten pro Jahr in Deutschland die zweithäufigste und in den USA mittlerweile die häufigste Todesursache in der männlichen Bevölkerung [1, 2]. Die Erkennung der Erkrankung in einem frühen Tumorstadium mit lokaler Begrenzung des Tumors ist unabdingbar für einen kurativen Therapieansatz. Mit Einführung der von den Krankenkassen getragenen Untersuchungsprogramme zur Früherkennung des Prostatakarzinoms 1972 wurde für Männer ab dem 45. Lebensjahr die digito-rektale Untersuchung der Prostata als Vorsorge empfohlen.

Die Ergebnisse dieses Früherkennungsprogramms waren enttäuschend. Bei 1,3 Millionen untersuchten Männern sind 1517 Patienten identifiziert worden, die ein Prostatakarzinom aufwiesen. Zudem wies die Mehrzahl der Patienten ein lokal fortgeschrittenes oder metastasiertes Stadium der Erkrankung auf [3]. Grundlegend änderte sich die Situation mit Einführung eines Tests für das prostataspezifische Antigen (PSA), welches erstmals durch Wang et al. 1979 [4] isoliert wurde. PSA wird in den Drüsenzellen der Prostata gebildet. Die Expression von PSA wird genetisch gesteuert und von Testosteron/Dihydrotestosteron stimuliert. Die physiologische Hauptfunktion von PSA besteht in der Verflüssigung des Ejakulates.

Im Rahmen der Diagnostik und Verlaufskontrolle muss berücksichtigt werden, dass pro Gramm Prostatagewebe $0,12-0,31 \mathrm{ng} / \mathrm{ml}$ PSA in das Serum abgegeben werden. Dies macht deutlich, dass es sich bei PSA nicht um einen tumorspezifischen, sondern um einen organspezifischen Marker handelt. In der Routineuntersuchung geht man derzeit von einem Schwellenwert von 4,0 ng/ml aus, der zukünftig wahrscheinlich auf 3,0 $\mathrm{ng} / \mathrm{ml}$ gesenkt wird.

Die Kombination von digito-rektaler Untersuchung der Prostata mit der PSA-Bestimmung und der Durchführung einer transrektalen Sonographie ermöglichen eine verbesserte Früherkennung, insbesondere kurativ behandelbarer Prostatakarzinome im Stadium pT1c/pT2 a/b, pN0, M0 [5]. Die aktuellen
Früherkennungsprogramme in den USA, den Niederlanden und auch Deutschland haben für die Behandlung des Prostatakarzinoms die wichtige Erkenntnis erbracht, dass Männer, die regelmäßig - also einmal pro Jahr und über mehrere Jahre - die Früherkennungsuntersuchungen nutzen, bei einer Erkrankung in mehr als $90 \%$ der Fälle exzellente Heilungsaussichten haben.

Statistiken zur Mortalität in Bezug auf das Prostatakarzinom haben in den USA erstmals den Beweis erbracht, dass durch Einführung eines Laborparameters die Sterblichkeit an einer Krebserkrankung gesenkt werden konnte [6].

Um in Deutschland die Effizienz der Früherkennung mit Hilfe der digito-rektalen Untersuchung und des PSA-Wertes zu prüfen, wurde 1997 unter Essener Leitung eine bundesweite Studie initiiert, in der 12000 Männer untersucht worden sind. Von 2338 auffälligen Befunden (suspekter Tastbefund und PSA-Wert über 4,0 ng/ml) konnten bislang 50\% durch eine Stanzbiopsie der Prostata weiter abgeklärt werden. In 262 Fällen wurde ein Prostatakarzinom gefunden. Dies entspricht einer Fallfindung von 3\%. Bei der Untersuchung des PSA-Wertes zeigt sich, dass besonders im Bereich von 4,0-10,0 ng/ml ein Tumorstadium vorliegt, das in mehr als $70 \%$ einem organbegrenzten Stadium entspricht, welches sicher und komplikationsarm kurativ behandelt werden kann [7]. Für die digito-rektale Untersuchung und die PSA-Bestimmung liegt der positiv-prädiktive Wert bei $50 \%$. Dies bedeutet, dass bei Männern mit einem PSA-Wert $\geq 4,0 \mathrm{ng} / \mathrm{ml}$, oder mit einem auffälligen Tastbefund eine Biopsie erfolgen sollte [8].

Somit hat das PSA nachhaltig die Diagnostik des Prostatakarzinoms beeinflusst. Ausdruck dessen ist, dass sich mit Einführung des PSA die histopathologische Klassifikation geändert hat. Das sogenannte klinische Stadium T1c umfasst Tumoren, die nicht bei der digito-rektalen Untersuchung getastet werden können, sondern durch eine nach Messung eines erhöhten

\begin{tabular}{ll}
\hline KARGER & $\odot$ 2002 S. Karger GmbH, Freiburg \\
Fax +497614520714 & Accessible online at: \\
$\begin{array}{l}\text { E-mail Information@Karger.de } \\
\text { www.karger.com }\end{array}$ & www.karger.com/journals/onk
\end{tabular}

Prof. Dr. Dr. h.c. Herbert Rübben

Universitätsklinikum Essen, Urologische Klinik und Poliklinik Hufelandstraße 55

D-45122 Essen (Deutschland)

Tel. +49 201 723-3211, Fax -5902

E-Mail herbert.ruebben@uni-essen.de 
PSA-Serumwertes durchgeführte Biopsie diagnostiziert wurden. Das Spektrum der T1c-Tumoren hinsichtlich der Ausbreitung und dem histopathologischen Stadium ist sehr weit. Hierunter fallen zum Teil große Tumoren, die durch ihre Lage in den vorderen Drüsenanteilen der Prostata nicht getastet werden können, ebenso jedoch kleine Tumoren, die trotz ihres geringen Tumorvolumens bereits zu einem Kapseldurchbruch geführt haben und die Umgebung infiltrieren können. Dies bedeutet, dass in der Gruppe der T1c-Karzinome solche Befunde sind, die keiner sofortigen Therapie bedürfen (insignifikante Tumoren) und andererseits T1c-Karzinome diagnostiziert werden, die ein Tumorvolumen über $0,5 \mathrm{~cm}^{3}$ aufweisen und somit als klinisch signifikant zu bezeichnen sind. Die Angaben, welches Tumorvolumen als signifikant angesehen werden muss, sind uneinheitlich und schwanken zwischen 0,2 und $0,5 \mathrm{~cm}^{3}$. Zur weiteren Abgrenzung insignifikanter T1c-Tumoren eignet sich ein auf der PSA-Bestimmung beruhendes Vorhersagemodell. Hier wird ein Verhältnis aus freiem und totalem PSA gebildet, wobei die Wahrscheinlichkeit für ein klinisch signifikantes Karzinom bei einem Anteil des freien PSA von $\leq 20 \%$ zunimmt. Keinen Einfluss auf den PSA-Wert hat eine vor Blutabnahme erfolgende rektale Untersuchung [6]. Kritisch zu bewerten ist die alleinige Verlaufsbeobachtung im Sinne der «Wait-and-watch-Strategie», die von der skandinavischen Gruppe um Johansson [9] favorisiert wurde. Im Rahmen einer nicht randomisierten Untersuchung dieser Gruppe zeigte sich bei alleiniger Verlaufsbeobachtung in 12,5 Jahren eine Krebssterblichkeit von nur 10\%. Aufgrund der vorgenommenen Selektionskriterien lässt die Studie jedoch keine verbindlichen Rückschlüsse zu. Demgegenüber konnte in der Untersuchung von Albertsen et al. 1998 [10] gezeigt werden, dass Patienten mit einem lokal begrenzten Karzinom und einem Gleason-Score von $\leq 4$ unter alleiniger Verlaufsbeobachtung nur ein minimales Risiko haben, innerhalb der nächsten 15 Jahre am Tumor zu versterben. Die Wait-and-watchStrategie ist jedoch kein aussichtsreiches Verfahren für Prostatakarzinome mit einem Gleason-Score von 7-10. Basierend auf den Untersuchungen von Albertsen et al. [10], ließ sich vielmehr die Lebenserwartung ausgedrückt in Jahren in Abhängigkeit vom Alter und dem Index der Begleiterkrankungen ermitteln. Dies erscheint insofern bedeutsam, als sich insbesondere beim lokal begrenzt wachsendem Prostatakarzinom aufgrund der Biologie der Erkrankung die Prognosen im Mittel nach 7,5 Jahren grundsätzlich unterscheiden, abhängig von der gewählten Behandlungsoption (Wait-and-watch-Strategie und radikale Prostatovesikulektomie oder Strahlentherapie). Voraussetzung zur Wahl des Therapieverfahrens ist somit nicht nur das lokale Tumorstadium, sondern eine geschätzte mittlere Lebenserwartung von mehr als 7 Jahren. Der Index der Begleiterkrankungen zur Ermittlung der geschätzten Lebenserwartung bei Patienten mit Prostatakarzinom beinhaltet die nachfolgenden Diagnosen:

- organische Herzerkrankung,

- koronare Herzkrankheit,
Tab. 1. Geschätzte Lebenserwartung in Abhängigkeit von Alter und dem Index der Begleiterkrankungen

\begin{tabular}{lrrr}
\hline Index & \multicolumn{3}{c}{ Alter bei Diagnosestellung, Jahre } \\
\cline { 2 - 4 } & 65 & 70 & \multicolumn{1}{c}{75} \\
\hline 0 & 17,9 & 14,8 & 11,9 \\
1 & 15,9 & 12,9 & 10,1 \\
2 & 10,8 & 8,4 & 6,3 \\
3 & 4,0 & 2,8 & 1,9 \\
durchschnittlich & 15,7 & 12,7 & 10,0 \\
\hline
\end{tabular}

- Herzrhythmusstörung,

- arterielle Hypertonie,

- Apoplex,

- arterielle Verschlusskrankheit,

- Diabetes mellitus,

- chronische Lungenerkrankung,

- Zweitmalignom,

- Lebererkrankung,

- Nierenerkrankung,

- Arthritis,

- Gastrointestinalerkrankung.

Basierend auf den geschilderten Begleiterkrankungen erfolgt die Bildung des Index:

Index 0: keine bekannten und zur Zeit existierenden Vorerkrankungen,

Index 1: asymptomatische oder therapeutisch gut behandelte Erkrankungen,

Index 2: behandelte, jedoch symptomatische Erkrankung, Index 3: unbehandelte, symptomatische Erkrankung.

In Abhängigkeit von dem so gebildeten Index beträgt die geschätzte Lebenserwartung eines zum Zeitpunkt der Diagnosestellung 65-jährigen Patienten zwischen 4,0 (Index 3) und 17,9 (Index 0) Jahren (Tab. 1).

Eine weitere Verbesserung einer stadiengerechten Diagnostik des Prostatakarzinoms zeichnet sich im Rahmen der Entschlüsselung des menschlichen Genoms ab. Dies führte zur Entwicklung neuer Technologien, die die Entschlüsselung von Genexpressionsprofilen molekularer Vorgänge im Gewebe sichtbar machen. DNA- und Tissue-Mikroarrays sind zwei neue Techniken, die auch in der onkologischen Forschung beim Prostatakarzinom bereits jetzt einen großen Stellenwert haben. Mit dem cDNA-Mikroarray (DNA-Chip) wird gleichzeitig das Genexpressionsprofil von mehr als 6000 Genen in einem Prostatagewebepräparat untersucht (Abb. 1). Diese Untersuchung liefert eine Darstellung der Über- oder Unterexpression von Genen in einem Prostatakarzinompräparat.

Die gezielte Prüfung der klinischen oder prognostischen Relevanz einzelner Gene und ihrer Produkte ist mit Hilfe einer komplementären Technik, dem sogenannten Tissue-Mikroarray möglich. Hierbei können einzelne identifizierte Gene an großen Kollektiven mit bis zu 400 Tumorentitäten an einem 


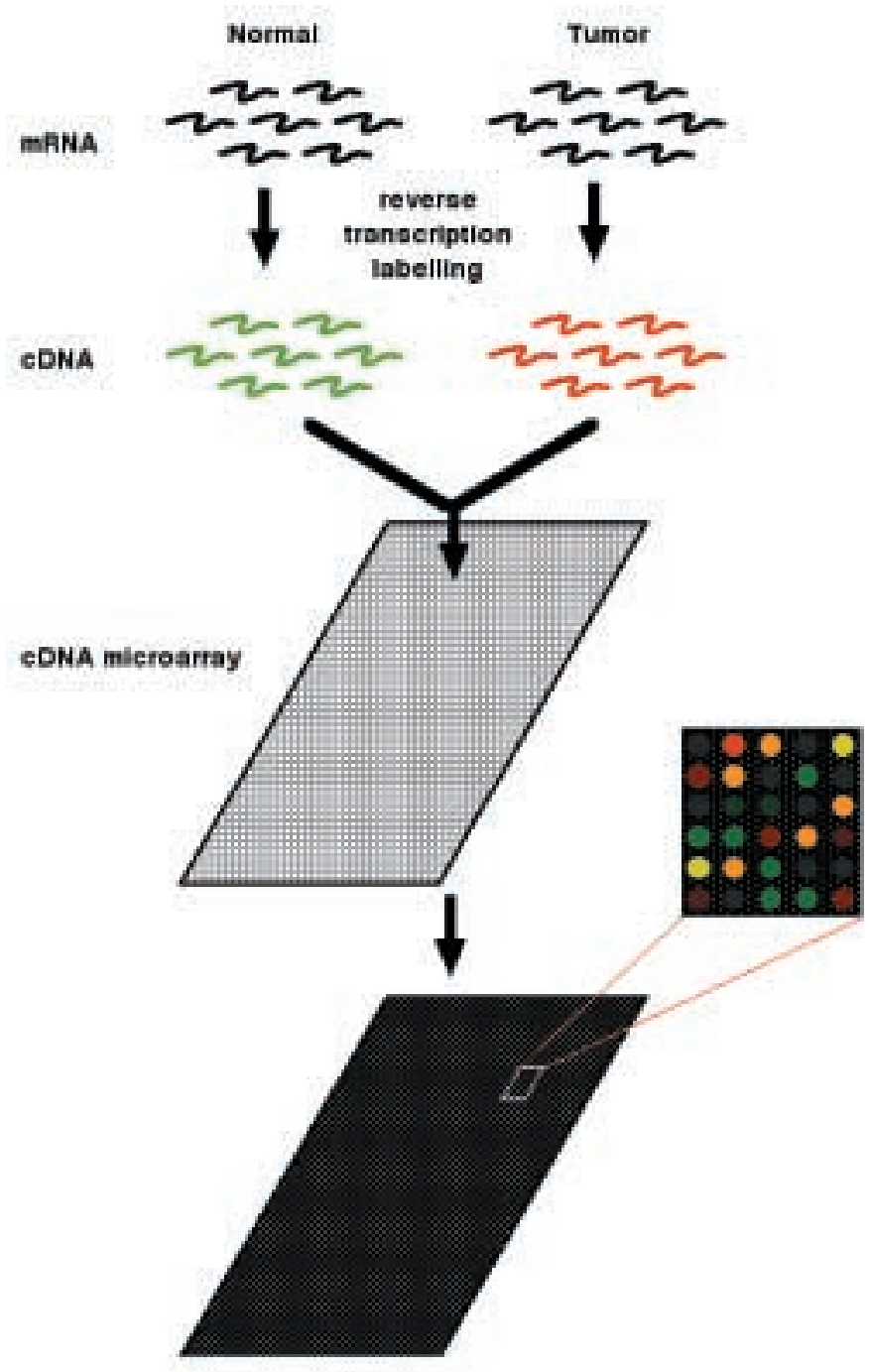

Abb. 1. cDNA-Mikroarray: Aus Tumor- und Normalgewebe wird mRNA extrahiert, durch reverse Transkription in cDNA umgeschrieben und auf den cDNA-Mikroarray aufgebracht. Im letzten Bild ist das Genexpresionsprofil dargestellt, das mit entsprechender Software analysiert wird.

einzigen Schnitt und in einem einzigen Arbeitsgang geprüft werden (Abb. 2). Erste Untersuchungen belegen auch für das Prostatakarzinom den sinnvollen Einsatz von DNA- und Tissue-Mikroarrays. Die Nutzung von Mikroarray-Technologien zur Aufdeckung molekularer Zusammenhänge und anschlieBender Prüfung auf deren klinische, prognostische und diagnostische Relevanz ist vergleichbar mit der Entwicklung des PSA-Tests.

Die Erkennung einer Prostatakarzinomerkrankung im frühen Stadium durch den Einsatz molekularer Marker ermöglicht nicht nur die Heilung, sondern beinhaltet insbesondere auch die Möglichkeit einer komplikationsarmen Operation. In diesem Zusammenhang wird angestrebt, den Kontinenzapparat (Musculus sphinkter externus) und die Funktion der Harnblase mit Erhalt des Blasenhalses, zu bewahren und durch nerv-
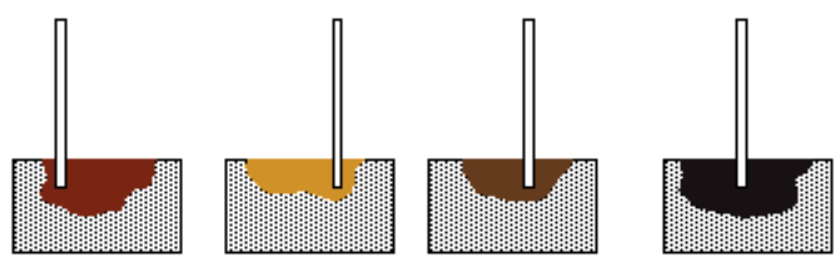

b
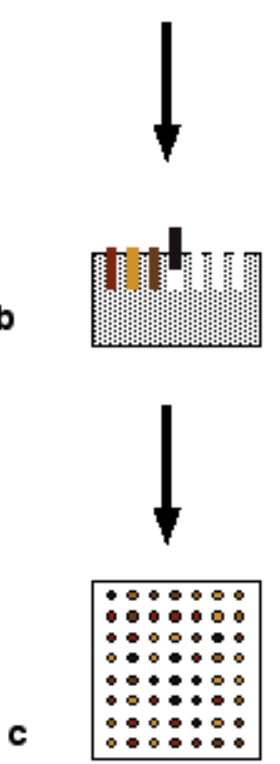

Abb. 2. Anfertigung eines Tissue Microarray: a Entnahme von Gewebeproben aus Paraffinblock mit spezieller Biopsienadel $(\varnothing=6 \mu \mathrm{m})$, b Einbringen der Gewebeproben in Tissue-Mikroarrayblock, c Schnitt von Tissue-Microarrayblock

schonende Operationsverfahren die erektile Funktion möglichst nicht zu beeinträchtigen. So hat die nervschonende Präparation bei Tumoren niedrigen Tumorstadiums $(\leq \mathrm{pT} 2 \mathrm{~b})$ die Rate einer Potenzerhaltung verfünffacht.

Eine weitere operative Option im Rahmen des Erhaltes der erektilen Funktion bietet sich durch die Verwendung autologer Nervinterponate an. Hier wird unter Verwendung von Anteilen des Nervus suralis ein bilaterales Interponat in den Fällen angestrebt, in denen aufgrund eines kapselüberschreitenden Tumorwachstums oder operationstechnischer Bedingungen eine Nervresektion erfolgte. Hier liegen erste Erfahrungen vor und belegen eine Wiedererlangung der erektilen Funktion nach 14-18 Monaten bei 75\% der Patienten [11].

Ein neu in die Diskussion gekommenes und bereits seit langem bestehendes Verfahren ist die interstitielle Radiotherapie im Sinne der Brachytherapie des Prostatakarzinoms. Hierbei wird die niedrigdosierte und die hochdosierte Brachytherapie unterschieden. Als Strahlenquelle für die niedrigdosierte Brachytherapie kommen ${ }^{125} \mathrm{I}$ oder ${ }^{103} \mathrm{Pd}$ zur Anwendung. Bei der hochdosierten Brachytherapie werden ${ }^{192} \mathrm{Ir}$ und ${ }^{198} \mathrm{Au}$ eingesetzt. Unterschieden werden permanente von temporären Strahlenquellen. Permanente Strahlenquellen sind ${ }^{125} \mathrm{I}$, 
${ }^{103} \mathrm{Pd}$ und ${ }^{198} \mathrm{Au}$. Als temporäre Strahlenquelle wird ${ }^{192} \mathrm{Ir}$ verwandt.

Im Gegensatz zu operativen Verfahren oder zur perkutanen Radiotherapie mit Dosen von 70-75 Gy findet sich für die Brachytherapie kein standardisiertes Vorgehen in Bezug auf die räumliche Verteilung des jeweiligen Strahlenträgers in der Prostata sowie dem konsekutiven PSA-Verlauf/Abfall nach Therapie [12]. Aufgrund dessen stellt die Brachytherapie ein bislang nicht ausreichend gesichertes alternatives Therapieverfahren dar, das im Rahmen von Studien geprüft werden sollte. Möglicherweise könnte sich eine künftige Indikation für Patienten ergeben mit einem niedrigen PSA-Ausgangswert (PSA $\leq 4 \mathrm{ng} / \mathrm{ml})$, einem niedrigen Tumorstadium $(\leq \mathrm{T} 2 \mathrm{a})$ und einem niedrigen Gleason-Score $(\leq 6)[13]$.

Nicht zu empfehlen ist die sogenannte Triple-Therapie des lokal begrenzten Prostatakarzinoms im Sinne einer kompletten Androgen-Blockade (LHRH-Analagon + Antiandrogen) mit Finasterid. Diese Therapie, bestehend aus 3 hormonell wirksamen Substanzen, erfolgt für den Zeitraum von 13 Monaten und wird danach nur noch mit Finasterid fortgeführt. Das von Leibowitz [14] auch in der Laienpresse propagierte Schema entspricht einer intermittierenden Androgendeprivation, wie sie in anderer Indikation bei metastasierten Erkrankungen zur Zeit im Rahmen internationaler Studien geprüft wird. Die Sinnhaftigkeit dieser Therapie beim lokal begrenz- ten Prostatakarzinom muss sehr kritisch bewertet werden, da die Hormontherapie eine Systembehandlung darstellt und der lokalen Erkrankung nicht gerecht wird. Dies kann bei zum Zeitpunkt der Erstdiagnose kurativ therapierbaren Tumoren zu einer Stadienverschlechterung und sogar zum Tod des Patienten führen. Zudem ist die Zugabe eines 5- $\alpha$-ReduktaseHemmers (Finasterid) zu einer kompletten Androgenblockade ohne wissenschaftliches Rational.

Weitere Fortschritte in der Behandlung des hormonrefraktären, symptomatischen Prostatakarzinoms zeichnen sich durch die Gabe von Zytostatika in Kombination mit Prednison ab. Hier führt die Applikation des Anthracendion-Derivats Mitoxantron zu einer nachhaltigen Verbesserung der Lebensqualität [15], wenn auch nicht der Überlebenszeit. Der Effekt der systemischen Chemotherapie ist auf den Anteil rasch proliferierender, refraktärer Prostatakarzinomzellen zurückzuführen, der weniger als 5\% der Tumorgesamtzellzahl ausmacht. Insofern erklärt sich das subjektive Ansprechen ohne erkennbare, objektiv messbare Remission. Fortschritte für die Lebensqualität der Patienten ergeben sich auch durch weitere Therapieoptionen, z.B. Therapie mit nichtsteroidalen Anabolika, die eine zytostatische Behandlung begleiten kann. Hierfür stehen Module wie der validierte Lebensqualitätsbogen der EORTC (QLQC30) zur Verfügung [5].

\section{Literatur}

1 Statistisches Bundesamt: Todesursachen in Deutschland. Stuttgart, Metzler-Poeschel, 1996, pp 26-34.

2 Waterbor J, Bueschen A: Prostate cancer screening (United States). Cancer Causes Control 1995;6: 267-274.

3 Flatten G: Krebsfrüherkennungsuntersuchung beim Prostatakarzinom - ein Erfahrungsbericht über 20 Jahre; in Faul P, Altwein J (eds): Screening des Prostatakarzinoms. Berlin, Springer, 1995, pp 33-40.

4 Wang MC, Murphy GP, Chu TM: Purification of a human prostate specific antigen. Invest Urol 1979; 17:159-163.

5 Schröder FH, Kranse R, Rietbergen J, Hoedemaeke R, Kirkels W: The European randomized study of screening for prostate cancer (ERSPC): An update. Members of the ERSPC, Section Rotterdam. Eur Urol 1999;35:539-43.
6 Altwein JE: Prostatakarzinom 169-232; in Rübben H (Hrsg): Uro-Onkologie. Heidelberg, Springer, 2001.

7 Luboldt HJ, Rübben H: Früherkennung des Prostatakarzinoms. Urologe A, 2000;39:22-26.

8 Luboldt HJ, Altwein JE, Bichler KH, Czaja D, Hüsing J, Fornara $P$, Jöckel $\mathrm{KH}$, Lübben $\mathrm{G}$, Schalkhäuser K, Weißbach L, Wirth M, Rübben H: Früherkennung des Prostatakarzinoms. Erste Ergebnisse einer prospektiven multi-zentrischen Studie in Deutschland. Urologe A 1999;38:114-123.

9 Johansson JE: Expectant management of early stage prostatic cancer. J Urol 1994;152:1753-1756.

10 Albertsen PC, Gleason DF: Competing risk analysis of men aged 55 to 74 years at diagnosis managed for clinically localized prostate cancer. JAMA 1998;280:975-980.
11 Kim E, Scardino PT: Bilateral nerve graft during radical retropubic prostatectomy: 1-year follow-up. J Urol 2001;165:1950-1956.

12 Otto T, Seegenschmidt H, Rübben H: Therapie des Prostatakarzinoms. MMW Fortschr Med 2000;142: 246-248.

13 Deger S, Böhmer D, Roigas J, Türk I, Budach V, Loening SA: Brachytherapie des lokalen Prostatakarzinoms. Urologe A 2001;40:181-184.

14 Leibowitz RL: Hormone blockade as the sole treatement of clinical stages T1-3 prostate cancer. Proc ASCO 2000;19:1493.

15 Tannock IF, Murphy KC: Chemotherapy with mitoxantrone plus prednisone or prednisone alone for symptomatic hormone-resistant prostate cancer. J Clin Oncol 1996;14:1756-1764. 